\title{
Research on Path Planning of Manipulator
}

\author{
Nana Wang ${ }^{1}$, Yizhun Peng ${ }^{1,2^{*}}$, Zhou Yang ${ }^{1}$, Yuheng Zhang ${ }^{1}$ \\ ${ }^{1}$ College of Electronic Information and Automation, \\ Tianjin University of Science and Technology, China; \\ ${ }^{2}$ Advanced Structural Integrity International Joint Research Centre, \\ Tianjin University of Science and Technology, China; \\ E-mail: *pengyizhun@tust.edu.cn \\ www.tust.edu.cn
}

\begin{abstract}
In order to further realize the flexibility, intelligence and human-machine friendliness, the robot must have the ability of flexible motion planning. This paper mainly introduces the general steps of manipulator path planning and common path planning algorithms. First, the path planning of the manipulator is reviewed from three aspects: environment modeling, path search, and path smoothing. In addition, compared various manipulator path planning algorithms, such as RRT algorithm, ant colony algorithm, genetic algorithm, etc.Finally, the development trend of path planning technology is summarized and analyzed.
\end{abstract}

Keywords: Manipulator, Path planning, RRT algorithm, Ant Colony Algorithm, Genetic Algorithms

\section{Introduction}

With the progress of the times, the application of robotic arm technology is becoming more and more popular. For the manipulator, reasonably planning its own movement path is the most basic function to complete the task in the real world, which is a key technology in robot research path planning. Path planning can be divided into two types: global path planning and local path planning. Global path planning refers to path planning based on all environmental information through certain algorithms under the condition that all environmental information is known. Local path planning refers to the situation where part or all of the environmental information is known.Real-time environmental information is collected by the sensor and the collected environmental information is analyzed for real-time path planning ${ }^{1}$. With the rapid development of the manipulator, the requirement for the path planning of the manipulator is getting higher and higher. Therefore, it is necessary to constantly optimize the path planning algorithm to save the movement time of the manipulator and reduce the joint loss of the manipulator.
This paper mainly introduces the path planning of 6-DOF manipulator.

\section{Path planning of 6-DOF manipulator}

Path planning should first establish an environment model that is convenient for computer to carry out path planning. Then, a walking path that enables the predicted performance function to obtain the optimal value is searched through a certain algorithm. Finally, the path searched through the corresponding algorithm is smoothen to make it a practical and feasible path ${ }^{2}$.

\subsection{Modeling Kinematics of manipulator}

\subsubsection{Modeling}

Aiming at the problem of how to describe the geometric parameters and coordinate system direction between the adjacent links of the mechanical arm, Denavit and Hartenberg came up with the DH method ${ }^{3}$, in this method, a coordinate system is fixed on each manipulator link, and the rotation and translation vectors are described by means of homogeneous transformation. The transformation 
relation from connecting rod $\mathrm{i}-1$ to $\mathrm{i}$ is shown in Equation (1). DH method is universally applicable. The joints between the link of the robot can be either rotating joints or prismatic joints, and there are no limits on the length and plane of the joints.

$$
\begin{aligned}
{ }_{i}^{i-1} T= & \operatorname{Rot}\left(x_{i-1}, \alpha_{i-1}\right) \operatorname{Trans}\left(x_{i-1}, \alpha_{i-1}\right) \\
& \operatorname{Rot}\left(z, \theta_{i}\right) \operatorname{Trans}\left(z, d_{i}\right)
\end{aligned}
$$

\subsubsection{Positive solution to the kinematic equation of the manipulator}

Forward kinematics is to find out the spatial position and attitude of the end-effector of the mechanical arm relative to the base coordinate system according to the Angle value of each joint of the manipulator and the parameter information of each lever ${ }^{4}$. The 6-DOF manipulator is composed of link connected with six joints, and the position and attitude of the end-effector relative to the base are formula $(2)^{5}$.

$$
{ }_{6}^{0} T={ }_{1}^{0} T_{2}^{1} T_{3}^{2} T_{4}^{3} T_{5}^{4} T_{6}^{5} T
$$

\subsubsection{Inverse solution to the kinematic equation of the manipulator}

Inverse kinematics refers to solving the Angle value of each joint of the manipulator based on the spatial position and attitude of the end-effector relative to the base coordinate system. Inverse transformation is often used to solve inverse kinematics, According to the forward kinematics equation of the manipulator, the solution process is formula (3). the Angle of joint 1 is calculated according to the corresponding matrix operation. Move the next joint Angle to the left of the equation and repeat until you have all the solutions.

$$
\left({ }_{1}^{0} T\right)^{-1}{ }_{6}^{0} T={ }_{2}^{1} T_{3}^{2} T_{4}^{3} T_{5}^{4} T_{6}^{5} T
$$

The inverse kinematics solution of the 6-DOF manipulator with the last three joints perpendicular to each other theoretically has a maximum of 8 sets, and then needs to be selected according to the actual joint Angle range 6 .

\subsection{Collision detection}

Collision detection technology is an important part of the path planning of the manipulator. It is mainly used to judge whether there is a collision between two objects. Accurate and effective collision detection method is the premise of effective obstacle avoidance path planning for the manipulator. Generally speaking, space obstacles are irregular geometric shapes. In order to judge the collision problem of manipulator conveniently, it is necessary to regularize the obstacles. Some scholars have proposed using the regular body envelope of obstacles to approximate modeling. Although this approximation expands the obstacle domain, it greatly simplifies the description of the obstacle domain and effectively improves the efficiency of planning ${ }^{7}$. Common obstacle envelopment models include: Sphere, AABB, OBB, KDOPS, etc.

Sphere is the smallest Sphere that contains objects ${ }^{8}$. This method is not only simple in structure, but also does not need to be updated in real time as the object rotates and moves. However, its tightness is poor and large redundancy space will be generated.So it's less used.

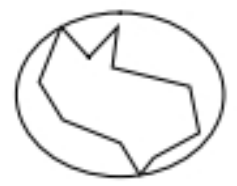

Fig. 1. This is a two-dimensional projection of sphere

$\mathrm{AABB}^{9-10}$ is the smallest hexahedron that contains an object and its sides are parallel to the axis. The AABB box model was the first to be used.This method is not only simple in construction but also fast in updating and relatively high in efficiency, which can be applied to the collision detection between deformed bodies.However, for some objects with irregular shape, its envelopment is poor, resulting in large redundancy space.

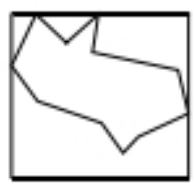

Fig. 2. This is a two-dimensional projection of $A A B B$ 
$\mathrm{OBB}^{11-13}$ is the smallest cuboid that contains an object and is arbitrary relative to the direction of the axes. OBB is a more commonly used type of bounding box.The biggest advantage of this method is its arbitrary orientation, which makes it possible to surround the object as closely as possible according to the shape characteristics of the surrounded object, but its construction is very difficult.

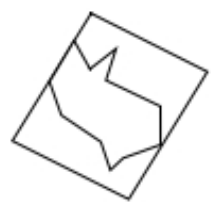

Fig. 3. This is a two-dimensional projection of OBB

$\mathrm{K}$-dop ${ }^{14-15}$ is a convex hull containing an object whose normal vector of all faces is taken from a fixed direction. It inherits the good compactness of convex hull and has the characteristics of simplicity ${ }^{16}$.

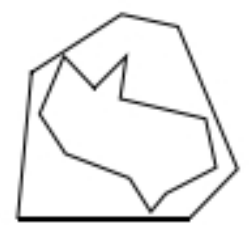

Fig. 4. This is a two-dimensional projection of K-DOP

\subsection{Environmental modeling}

The actual working environment of mobile robot is a real physical space, while the space handled by path planning algorithm is the abstract space of the environment.Environment modeling is a mapping from physical space to abstract space. This efficient description of the robot's active space is called environment modeling ${ }^{17}$. Environment model is the basis of path planning algorithm, whether it can fully reflect the environment information and establish environment model effectively is related to the performance of the whole path planning algorithm. When the environment information is fully known, the global environment model can be established.In practical applications, the working environment of mobile robots is often uncertain, which makes it impossible for robots to build global environment model in advance, but to build local environment model in real time according to sensor information ${ }^{18}$.

Environment modeling is a very important part of robot path planning. The reasonable environment model is beneficial to the reduction of search volume and the saving of space-time cost in the path planning process. Common environment representation methods include raster method, cell tree method and polygon representation method.

\subsection{Path search}

The path searching stage is to apply the corresponding algorithm to find a walking path based on the environment model so that the predetermined performance function can get the optimal value. Common path search algorithms include RRT algorithm, ant colony algorithm, genetic algorithm, etc.

\subsubsection{RRT algorithm}

RRT algorithm is a global path planning algorithm based on random sampling proposed by Steven M. aValle. This method does not need to be learned, and is suitable for path planning in dynamic environment. RRT algorithm can be divided into two categories, one of which is Single directional rapidly-exploring random tree (Single-RRT), and the other is Bi-directional rapidly-exploring random tree (BI-RRT). The single-RRT method randomly selects a point in the moving space of the manipulator as a node in the tree, and continuously searches for nodes to expand the tree until a path is found from the starting point to the target point. The idea of BI-RRT algorithm is to generate two fast search random trees from the starting point and the target point until the two trees are connected. The advantage of this algorithm is that the two trees can be expanded in parallel. The complexity of this algorithm lies in how to make the two trees cross connected. The following mainly introduces the single branch search rapid expansion of random number method, the main process is as follows ${ }^{19}$.

(1) The starting point Xstart and target point Xgoal of the manipulator are given in the working environment space, and the starting point is taken as the target point of the growth tree.

(2) The search step of the given algorithm is L0, and a random tree Trand in the manipulator workspace with $\mathrm{N}$ nodes is generated from the starting point Xstart.

(3) Select any random point Xrand in the manipulator workspace.

(4) Traverse the random tree Trand, calculate the distance from each node of Trand to the random point Xrand, and screen out the point Xnear closest to the random point Xrand among the $\mathrm{N}$ nodes.

(5) Take the line of Xnear and random point Xrand as the growth direction, take Xnear as the starting point, and generate a new node Xnew along the growth direction with the step length of the algorithm as the length. 
(6) Judge whether Xnew has collision with the obstacle. If there is no collision, add it to the random tree Trand; if there is collision, discard the node Xnew.

(7) Judge whether the distance between Xnew and Xgoal is less than the setting neighborhood (DISfinish) of the end point.If less than, the path search ends, if not less than repeat steps 3-6 until less than.

The advantage of RRT algorithm is that it has powerful ability to search unknown space, and it is often used for path navigation in unknown environment, and it is fast.However, the planned path is not smooth enough. When the sampling domain of RTT algorithm and the original problem are not suitable, the convergence rate of standard RRT algorithm will be slow.

\subsubsection{Ant Colony Algorithm}

$\mathrm{ACA}^{20}$ is a heuristic global path planning algorithm proposed by Italian scholar Dorigo Mden. The main idea of this algorithm is that each ant leaves a certain concentration of pheromone on the path it travels while foraging, and the concentration of pheromone is inversely proportional to the length of the path. By adopting the positive feedback mechanism, the shortest path with a high concentration of pheromone will be found soon. The basic steps of the ant colony algorithm are as follows(Take environment modeling as a raster method as an example $)^{21}$ :

(1)Set the initial grid of path planning as Sstart and the target grid as Sgoal.The initialization cycle number Nstart is 0 , the maximum cycle number is Nmax, the information heuristic factor is $\alpha$, the expected heuristic factor is $\beta$, the pheromone volatility coefficient $\rho$, constant $Q$, and the initial pheromone on the path between grids is constant $\tau_{i j}(0)$

$=$ const.M ants were placed at the starting point of the path planning, Sstart, and Sstart was added to the tabu. (2)If the current grid is not the target grid, select the next grid according to the probability calculated by the formula below, and add the selected grid to your own tabu list. The probability that ant $\mathrm{K}$ at node $\mathrm{I}$ chooses node $\mathrm{J}$ as the transfer direction is formula (4).

$$
\begin{aligned}
& P_{i j}^{\mathrm{k}}(t)=\frac{\tau_{i j}^{\alpha}(t) \eta_{i j}^{\beta}(t)}{\sum_{s \in \text { allowed }_{k}} \tau_{i j}^{\alpha}(t) \eta_{i s}^{\beta}(t)}, j=\text { allowed }_{k} \\
& P_{i j}^{\mathrm{k}}(t)=0, j=\text { otherwise }^{2}
\end{aligned}
$$

(3)Repeat execution 2 until all ants have completed the subsequent grid selection.

(4)Repeat 2,3.Until all ants have moved to the target grid.
(5)By counting the total length of the path taken by each ant, the shortest path and the length of the shortest path in this cycle are obtained.

(6)Update the pheromone concentration on each path according to Formula (5).

$$
\begin{aligned}
& \tau_{\mathrm{ij}}(t+n)=\rho^{*} \tau_{i j}(t)+\Delta \tau_{i j}, \rho \in(0,1) \\
& \Delta \tau_{i j}=\sum_{k=1}^{m} \Delta \tau_{i j}^{k}
\end{aligned}
$$

(7)Tabu table cleared, cycle times increased by 1 .If the number of cycles is less than the maximum number of cycles, go to Step 2; otherwise, skip the entire cycle and output the optimal path and the optimal path length.

This algorithm is a distributed system, which has strong adaptive ability and good global optimization ability, but it has a large amount of computation, is easy to fall into the local optimal solution, and does not have real-time ability.

\subsubsection{Genetic Algorithms}

$\mathrm{GA}^{22}$ is an iterative search method based on the principle of genetics proposed by John Holland in 1962. The algorithm is mainly composed of eight steps, respectively set up maps, initialization of population, calculating the value of individual fitness, choosing the appropriate individuals into the next generation, crossover, mutation, update the population, if appear the optimal path or the number of iterations is output optimal solution, otherwise, continue to iteration, the optimal output of the individual as the optimal solution.

The method requires fewer objective functions and constraints, and the optimization scope is extended to the whole independent variable space. After iteration, the method can approach the global optimal solution with greater probability.At the same time, genetic algorithm can solve nonlinear and complex optimal solutions effectively by using random transformation rules instead of deterministic rules. Its greatest advantage is that it is easy to combine with other algorithms, and it gives full play to its iterative advantages.However, due to the large storage space and long operation time of this algorithm, the operation efficiency is not high and it is easy to converge too early.

\subsection{Path smooth}

In general, the path planned by path search algorithm is a continuous line segment composed of some discrete points, and the shape of the path is not smooth. This not only reduces the motion stability of the mechanical arm, but also causes the impact and 
vibration of the motor and accelerates the wear of the mechanical parts.Therefore, path smoothing is very important to ensure the smooth movement of the manipulator.Quadric B-spline curves are often used to smooth obstacle avoidance paths so that the manipulator can work continuously and stably.

\section{The conclusion}

As the robotic arm is more and more widely used in various fields, the path planning environment of the robotic arm is more complex and changeable.This requires that the path planning algorithm should have the ability to respond quickly to complex environment changes. This paper summarizes the basic methods of path planning.In the future, path planning technology will be applied in more and more fields, and more efficient, flexible and accurate path planning algorithms will be born.

\section{References}

1. Chen Gu,Study on modular manipulator design and its path planning method. jiangsu,Jiangsu University of Science and Technology,2014.

2. Guanglin Zhang,Xiaomei Hu,Summary of path planning algorithm and its application.The modern machinery, 2011.

3. Zhaoguang Wang,Rrsearch on obstacle avoidance path planning for 6-DOF manipultor. Southwest Jiaotong University, 2018.

4. Chengxing Zong,Research on obstacle avoidance motion planning method for spatial multi DOF serial manipulator.Hefei ,Hefei university of Technology,2017.

5. Guoxiu Cheng,Research of Obstacle avoidance path planning based on manipulator with six degrees of freedom.Shenyang, Northeastern University,2012.

6. WeiSheng, The research of planning technology for obstacle avoidance path based on multi-degree of freedom industrial manipulator.Jiangsu, Jiangnan university, 2012.

7. Qingxuan Jia,Gang Chen,Path planning for space manipulator to avoid obstacle based on $\mathrm{A}^{*}$ algorithun. journal of mechanical end ineering,2010,46(13).

8. Guangmiao Jiang,Research and application of collision detection algorithm based on bounding box.University of Electronic Science and Technology,2012.

9. Xuewen Chen,Wusheng Chou, Research on collision detection algorithm based on bounding box.Computer Engineering and applications, 2005,41(5):46-50.

10. Shaowei Fan,Research on real-time collision detection technology. Zhejiang university, 2003.

11. Zhilei Ding, Fast collision detection based on OBB bounding box. Lanzhou University of Technology,2006.

12. Gottschalk S.Collision queries using oriented bounding boxes:The University of North Carolina at Chapel Hill,2000.

13. D.Baraff. Interactive simulation of solid rigid bodies. IEEE Computer Graphics and Applications, 1995, 15(3):6375

14. Kim Y J, Lin M, Manocha D. Fast penetration depth estimation using rasterization hardware and hierarchical refinement.Proc of Symposium on Computational Geometry.2003:386-387

15. Klosowski J, Held M, Mitchell J S B,etal. Efficient collision detection using bounding volume hierarchies of $\mathrm{k} 2$ 
DOPs. IEEE Transaction on Visualization and Computer Graphics, 1998,4(1):21-37

16. Tao Wang, The Study of robotic path planning and related simulation system based on ant algorithm.Xian,Xi an University of Science and Technology,2009.

17. Lihui Peng,Research on the kinematics and path planning of manipulator.Hunan,Hunan University of Technology,2012.

18. Chao Ma,Research on path Planning of $6 \mathrm{R}$ tandem manipulator in complex space environment.Jinan University, 2017.

19. Jinming Dong, Research on path Planning based on Ant colony algorithm.Xian,Shaanxi Normal University, 2009.

20. Jincai Ma,Research on path planning based on six-DOF manipulator.South China University of Technology, Guangzhou.2013

21. J Tu, S Yang. Genetic Algorithm Based Path Planning for a Mobile Robot.Taiwan: Proceedings of IEEE Intelligent Conference on Robotics and Automation,3003:P1221-P1226.

22. Tao Han,Huaiyu Wu,Research on path planning for manipulator to avoid real-time obstacle based on genetic algorithms.Applicatian Research f Camputers, 30(5),2013. 